\title{
Reprogramming of arachidonate metabolism confers drug resistance to glioblastoma through Enhancing Mitochondrial Activity in Fatty Acid Oxidation
}

\author{
Yu-Ting Tsai \\ Taipei Medical University \\ Wei-Lun Lo \\ Taipei Medical University \\ Pin-Yuan Chen \\ Keelung Chang Gung Memorial Hospital of the CGMF
}

Chiung-Yuan Ko

Taipei Medical University

\section{Tzu-Jen Kao}

Taipei Medical University

\section{Wen-Bing Yang}

Taipei Medical University

\section{Kwang-Yu Chang}

National Health Research Institutes

\section{Chia-Yang Hung}

Beckman Research Institute City of Hope: City of Hope Beckman Research Institute

\section{Ushio Kikkawa}

Taipei Medical University

\section{Wen-Chang Chang}

Taipei Medical University

\section{Tsung-I Hsu ( $\sim$ dabiemhsu@tmu.edu.tw)}

Taipei Medical University https://orcid.org/0000-0002-7524-7740

\section{Research}

Keywords: TMZ-resistant GBM, Sp1, PGE2, Mitochondria, fatty acid $\beta$-oxidation

Posted Date: October 18th, 2021

DOI: https://doi.org/10.21203/rs.3.rs-965026/v1 
License: (c) (i) This work is licensed under a Creative Commons Attribution 4.0 International License. Read Full License 


\section{Abstract \\ Background}

Sp1 is involved in the recurrence of glioblastoma (GBM) due to the acquirement of resistance to temozolomide (TMZ). Particularly, the role of Sp1 in metabolic reprogramming for drug resistance remains unknown.

\section{Methods}

RNA-Seq and mass spectrometry were used to analyze gene expression and metabolites amounts in paired GBM specimens (primary vs. recurrent) and in paired GBM cells (sensitive vs. resistant). $\omega-3 / 6$ fatty acid and arachidonic acid (AA) metabolism in GBM patients were analyzed by targeted metabolome. Mitochondrial functions were determined by Seahorse XF Mito Stress Test, RNA-Seq, metabolome and substrate utilization for producing ATP. Therapeutic options targeting prostaglandin (PG) E2 in TMZ-resistant GBM were validated in vitro and in vivo.

\section{Results}

Among the metabolic pathways, Sp1 increased the prostaglandin-endoperoxide synthase 2 expression and PGE2 production in TMZ-resistant GBM. Mitochondrial genes and metabolites were obviously increased by PGE2, and these characteristics were required for developing resistance in GBM cells. For inducing TMZ resistance, PGE2 activated mitochondrial functions, including fatty acid $\beta$-oxidation (FAO) and tricarboxylic acid (TCA) cycle progression, through PGE2 receptors, E-type prostanoid (EP) 1 and EP3. Additionally, EP1 antagonist ONO-8713 inhibited the survival of TMZ-resistant GBM synergistically with TMZ.

\section{Conclusion}

Sp1-regulated PGE2 production activates FAO and TCA cycle in mitochondria, through EP1 and EP3 receptors, resulting in TMZ resistance in GBM. These results will provide us a new strategy to attenuate drug resistance or to re-sensitize recurred GBM.

\section{Background}

Glioblastoma (GBM), which is classified as a grade IV glioma, is the most aggressive and malignant brain tumor.[1] Its current standard treatment is tumour resection followed by chemotherapy and radiotherapy, and in this regard, temozolomide (TMZ), an oral alkylating agent, is the most widely used chemotherapy agent. However, with this current standard treatment, GBM still remains incurable and is characterized by a high prevalence of recurrence owing to its acquisition TMZ resistance.[1] 
Previously, we reported that specificity protein 1 (Sp1) promotes the development of malignant characteristics in GBM, including drug resistance and cancer stem cell enrichment.[2-8] Particularly, it enhances resistance to TMZ by upregulating the expression of cytochrome p450 (CYP) 17A1, which catalyses the metabolism of cholesterol to neurosteroids. $[4,5,9,10]$ Therefore, these findings suggest that $\mathrm{Sp} 1$ plays an important role in the acquisition of drug resistance in cancers by regulating the reprogramming of arachidonate metabolism. Therefore, in this study, our objective was to clarify the role of $\mathrm{Sp} 1$ in regulating arachidonate metabolism, leading to GBM drug resistance.

With respect to phospholipid-prostaglandin metabolism, prostaglandin E2 (PGE2) is upregulated and is frequently observed in various types of cancer.[11-13] Specifically, in GBM, the enzyme that is primarily responsible for PGE2 synthesis, cyclooxygenase-2 (COX2)/prostaglandin-endoperoxide synthase 2 (PTGS2), reportedly promotes cancer cell proliferation and migration, $[14,15]$ suggesting that COX2 enhances arachidonic acid (AA) metabolism, which is necessary for the survival of GBM cells, to synthesize PGE2. However, the correlation between AA-derived metabolites and drug resistance in GBM remains unclear.

To sustain rapid proliferation, cancer cells take advantage of the reprograming of metabolic networks to generate sufficient bioenergy to support their cellular functions. Even though it is well known that the Warburg effect is the metabolic reprogramming that enhances aerobic glycolysis for cancer proliferation, [16] the importance of mitochondria-mediated metabolism has been raised recently to challenge the Warburg effect in GBM. Evidence suggests that GBM cells rely on oxidative phosphorylation in mitochondria to generate bioenergy.[17, 18] Additionally, fatty acid $\beta$-oxidation (FAO), which is the main step in lipid metabolism for the generation of bioenergy inside the mitochondria, has also been considered to play an important role in tumour development in nutrient-deprived environments[18] and cancer metastasis.[19, 20] However, its role in cancer drug resistance is still unknown.

In this study, we observed that Sp1 enhances the synthesis of PGE2 from AA in patients with recurrent GBM, and that PGE2 induces TMZ resistance by enhancing mitochondrial activity. Our findings provide a novel mechanism for the reprograming of AA metabolism to the end of combating drug resistance. They also suggest the possibility of realizing GBM treatment based on the combination of the EP1 antagonist, ONO-8713, and TMZ to reverse drug resistance.

\section{Materials And Methods}

\section{Human samples}

GBM samples were obtained from patients with GBM admitted to the Keelung Chang Gung Memorial Hospital, Linkou Chang Gung Memorial Hospital, and Taipei Medical University-Shuang-Ho Hospital. The characteristics of these patients are summarized in Supplementary Tables S1 and S2 and Supplementary Fig. 1 The pathologies of the human brain tumour samples were determined according to WHO classification.[21, 22] Isocitrate dehydrogenase 1 (IDH-1), glial fibrillary acidic protein (GFAP), and $0^{6}$ - 
methylguanine-DNA methyltransferase (MGMT) were used as the diagnostic and prognostic makers for GBM. $[23,24]$

\section{Cell culture}

GBM cell lines U87MG, A172, and T98G were purchased from American Type Culture Collection (ATCC, Manassas, VA, USA). Further, Pt\#3 and Pt\#5 were isolated from patients with GBM, and P1S was obtained from another patient, who exhibited therapeutic resistance right from the onset of treatment.[5] The cells were cultured in Dulbecco's Modified Eagle Medium (DMEM, Thermo Fisher Scientific, Waltham, MA, USA) supplemented with $10 \%$ foetal bovine serum (GE Healthcare Life Sciences, South Logan, UT, USA), $100 \mu \mathrm{g} / \mathrm{mL}$ penicillin, and $100 \mu \mathrm{g} / \mathrm{mL}$ streptomycin (Thermo Fisher Scientific) at $37^{\circ} \mathrm{C}$ in a $5 \%-\mathrm{CO}_{2}$ incubator. TMZ-resistant U87MG-R and Pt\#3-R cells were established via long-term treatment with TMZ as described previously.[2, 4]

\section{Establishment of Sp1 knockout cells}

Sp1 knockout cells were prepared using clustered regularly interspaced short palindromic repeats (CRISPR) strategy under the assistance of Biotools Co., Ltd. (New Taipei City, Taiwan). Expression vectors for guide RNA (U6-gRNA) and Cas9 gene (CMV-p-Cas9), which are ampicillin-resistant, were obtained using the Escherichia coli strain DH5a as a host. Surrogate reporter vectors, which are kanamycin resistant, were also purified from the transformed E. coli. Cells were transfected with plasmids by using Lipofectamine ${ }^{\circledR}$ LTX \& PLUS ${ }^{\text {TM }}$ Reagent (15338-100, Thermo Fisher Scientific). Briefly, $10 \mu \mathrm{L}$ of LTX Reagent were diluted in $100 \mu \mathrm{L}$ of Opti-MEM Medium (Thermo Fisher Scientific), and $2.5 \mu \mathrm{g}$ of plasmid DNA (Cas9: sgRNA: surrogate $=1: 1: 0.5$ ) were diluted in $100 \mu \mathrm{L}$ of Opti-MEM Medium. sgRNA was designed: 5'-AGGAGTTGGTGGCAATAATGGGG-3'. After hygromycin selection, Sp1-knockout cell colonies were identified and the depletion was confirmed by Western blotting.

\section{Chemicals}

Chemicals in Supplementary Table 3 were dissolved in the appropriate solvent according to the manufacturers' instruction for stock solutions. Other materials were described in the respective paragraphs in this section. EP1, EP3, and EP4 antagonists were provided by Ono Pharmaceutical Co., Ltd (Osaka, Japan).

\section{Chemicals and reagents for targeted metabolomics}

All eicosanoids and deuterated IS were purchased from Cayman Chemical (Ann Arbor, MI, USA). HPLCgrade acetonitrile ( $\mathrm{ACN})$ and methanol $(\mathrm{MeOH})$ were purchased from Merck (Darmstadt, Germany). MilliQ water (Millipore, Bradford, USA) was used in all experiments. Acetic acid was purchased from SigmaAldrich. CNW Poly-Sery MAX SPE cartridges were from ANPEL Co. (Shanghai, PRC). The stock solutions of standards were prepared at the concentration of $0.1 \mathrm{mg} / \mathrm{mL}$ in $\mathrm{MeOH}$. All stock solutions were stored at $-20^{\circ} \mathrm{C}$. The stock solutions were diluted with $\mathrm{MeOH}$ to working solutions before analysis.

\section{Sample preparation for targeted metabolomics}


Tissue samples (about $50 \mathrm{mg}$ ) and $10 \mu \mathrm{L}$ of $\mathrm{BHT}$ (butylated hydroxytoluene)/MeOH solution (W/V, 4.8 $\mathrm{g} / 100 \mathrm{~mL}$ ) were subjected to protein precipitation by adding $100 \mu \mathrm{L}$ of $\mathrm{MeOH}$ containing deuteriumlabeled IS, at a final concentration of $50 \mathrm{ng} / \mathrm{mL}$ each of PGE2-d4, 6-keto PGF1a-d4, 5-HETE-d8 and 100 $\mathrm{ng} / \mathrm{mL}$ of $9-\mathrm{HODE}-\mathrm{d} 4$. Samples were extracted with $500 \mu \mathrm{L}$ of $\mathrm{MeOH}$ using a tissuelyzer at $50 \mathrm{~Hz}$ for 30 sec (3 times) followed with 5 ultrasonication cycles ( 1 min treatment and $1 \mathrm{~min}$ break). The supernatant was transferred to new tubes after centrifuged at $12000 \mathrm{rpm}$ for $10 \mathrm{~min}$ at $4^{\circ} \mathrm{C}$, and followed by diluting with pure water to $15 \% \mathrm{MeOH}$ concentration, followed by solid phase extraction (SPE) pretreated with $\mathrm{MeOH}$ and equilibrated with $\mathrm{H} 2 \mathrm{O}$. The extract was dried and then re-dissolved in $100 \mu \mathrm{L}$ of $\mathrm{MeOH}$, followed by filtering the solution with a $0.22 \mu \mathrm{m}$ membrane filter before UPLC-MS/MS analysis.

\section{Liquid chromatography and mass spectrometry}

UPLC-MS/MS analyses were conducted on an Agilent UPLC-MS/MS system consisting of 1290 UPLCsystem coupled with an Agilent 6470 triple-quadrupole mass spectrometer (Agilent Technologies). For analysis, $3 \mu \mathrm{L}$ of the extract were injected. Chromatographic separation was achieved on an Agilent ZORBAX RRHD Eclipse XDB C18 column $(2.1 \times 100 \mathrm{~mm}, 1.8 \mu \mathrm{m}$ particles $)$ using a flow rate of 0.659 $\mathrm{mL} / \mathrm{min}$ at $45^{\circ} \mathrm{C}$ during a 13 min gradient (0-12 min from $68 \% \mathrm{~A}$ to $20 \% \mathrm{~A}, 12-13$ min $5 \% \mathrm{~A}$ ), while using the solvents $A$, water containing $0.005 \%$ formic acid, and $B$, acetonitrile containing $0.005 \%$ formic acid. Electrospray ionization was performed in the negative ion mode using N2 at a pressure of $30 \mathrm{psi}$ for the nebulizer with a flow of $10 \mathrm{~L} / \mathrm{min}$ and a temperature of $300^{\circ} \mathrm{C}$, respectively. The sheath gas temperature was $350^{\circ} \mathrm{C}$ with a flow rate of $11 \mathrm{~L} / \mathrm{min}$. The capillary was set at $3500 \mathrm{~V}$ and the nozzle voltage was 500 $\mathrm{V}$. Multiple reaction monitoring (MRM) has been used for quantification of screening fragment ions.

Data preprocessing: Peak determination and peak area integration were performed with MassHunter Workstation software (Version B.08.00, Agilent Technologies) while auto-integration was manually inspected and corrected if necessary. The obtained peak areas of targets were corrected by appropriate IS and calculated response ratios were used throughout the analysis.

\section{Untargeted metabolomics}

The ultra-high performance liquid chromatography-quadrupole time-of-flight mass spectrometry (UHPLCQ-TOF-MS) analysis was performed using an UHPLC system (1290, Agilent Technologies, Santa Clara, CA, USA) with a UPLC BEH Amide column $(1.7 \mu \mathrm{m} 2.1 * 100 \mathrm{~mm}$, Waters, Milford, MA, USA) coupled to TripleTOF 6600 (Q-TOF, AB Sciex, Framingham, MA, USA). The mobile phase consisted of $25 \mathrm{mM}$ $\mathrm{NH} 40 \mathrm{Oc}$ and $25 \mathrm{mM} \mathrm{NH} 4 \mathrm{OH}$ in water $(\mathrm{pH}=9.75)(\mathrm{A})$ and acetonitrile $(\mathrm{B})$ were carried with elution gradient as follows: 0 min, 95\% B; 7 min, 65\% B; 9 min, 40\% B; 9.1 min, 95\% B; 12 min, 95\% B, which was delivered at $0.5 \mathrm{ml}$ per min. The injection volume was $2 \mu \mathrm{L}$. The Triple TOF mass spectrometer was used for its ability to acquire MS/MS spectra on an information dependent basis (IDA) during an LC/MS experiment. In this mode, the acquisition software (Analyst TF 1.7, AB Sciex) continuously evaluates the full scan survey MS data as it collects and triggers the acquisition of MS/MS spectra depending on preselected criteria. In each cycle, 12 precursor ions whose intensity greater than 100 were chosen for fragmentation at collision energy (CE) of $30 \mathrm{~V}$ (15 MS/MS events with product ion accumulation time of $50 \mathrm{msec}$ each). 
ESI source conditions were set as following: lon source gas 1 as 60 Psi, lon source gas 2 as 60 Psi, Curtain gas as $35 \mathrm{Psi}$, source temperature $650^{\circ} \mathrm{C}$, lon Spray Voltage Floating (ISVF) $5000 \mathrm{~V}$ or $-4000 \mathrm{~V}$ in positive or negative modes, respectively.

\section{Enzyme-linked immunosorbent assay (ELISA)}

AA and PGE2 in culture media of wild type and TMZ-resistant U87MG cells were determined using Arachidonic Acid ELISA Kit (E4602, Biovision, Milpitas, CA, USA) and Prostaglandin E2 ELISA Kit (514010, Cayman, Ann Arbor, MI, USA), respectively, according to the manufacturers' instruction.

\section{Promoter reporter assay}

Plasmids containing each promoter region were transfected into targeted cell lines. Cells were harvested with diluted Cell Culture lysis 5X Reagent (Promega, San Luis Obispo, CA, USA). $10 \mu \mathrm{L}$ of sample were then mixed with $10 \mu \mathrm{L}$ of luciferin (Promega). Luminometer (HIDEX, Tampa, FL, USA) was used to measure the promoter activity of the mixture.

\section{3- (4,5-dimethylthiazol-2-yl)-2,5-diphenyltetrazolium bromide (MTT) assay}

Cells $\left(2 \times 10^{4}\right.$ cells/well in a 24-well plate) were treated with different doses of drugs for 4 days. After the treatment, $300 \mu \mathrm{L}$ of fresh medium containing $0.5 \mathrm{mg} / \mathrm{mL}$ MTT reagent (Sigma-Aldrich, St. Louis, MO, USA) were added to each well and incubated for $15 \mathrm{~min}$ at $37^{\circ} \mathrm{C}$. Medium was removed and the crystals were dissolved in $300 \mu \mathrm{L}$ of DMSO (Sigma-Aldrich). The absorbance was measured at $550 \mathrm{~nm}$ by using an iMark Microplate Absorbance Reader (Bio-Rad, Hercules, CA, USA).

\section{Transmission electron microscopy (TEM)}

To prepare the sample for TEM, cells were washed by PBS and fixed in $2 \%$ paraformaldehyde, $2.5 \%$ glutaraldehyde, and $0.1 \mathrm{M}$ cacodylate (MERCK Millipore, Billerica, MA, USA) at room temperature. The samples were then sent to Taipei Medical University Core Facility (Taipei, Taiwan) for further preparation and imaging.

\section{RNA-Seq and bioinformatics}

After total RNA extraction, samples were subjected to genomic sequencing. The gene expression influenced by Sp1 for 1.5 folds was sorted, and the functional grouping was performed using the Ingenuity Pathway Analysis (IPA) system

(https://www.qiagenbioinformatics.com/products/ingenuitypathway-analysis). Metabolism genes were sorted using Cancer Cell Metabolism Genes [25]. Heatmaps were prepared based on the level of expression using ToppCluster (https://toppcluster.cchmc.org/).

\section{Chromatin immunoprecipitation coupled with sequencing (ChIP-Seq)}


U87MG cells were fixed with $1 \%$ formaldehyde for preserving the protein-DNA interactions, and DNAprotein complexes were harvested using the Simple ChIP enzymatic chromatin IP kit (\#9003, Cell Signaling Technology, Danvers, MA, USA) followed by NextSeq 500 high throughput sequencing system (Illumina, San Diego, CA, USA) as described previously [7, 26]. XFe24 Seahorse Mitochondrial Respiration Mito Stress Test

Cells were treated with different conditions of TMZ, PGE2 or EP1-EP4 antagonists for 4 days. After the treatment, cells were trypsinized and $2 \times 10^{4}$ cells/well were seeded into the XFe24 Cell Culture Microplates (Agilent Technologies) and incubated for a day. Meanwhile, a sensor cartridge (detecting probes, Agilent Technologies) in Seahorse XF Calibrant at $37^{\circ} \mathrm{C}$ was hydrated in a non-CO2 incubator overnight for the following experiments. In the assay day, the cell-cultured medium was replaced with assay medium (DMEM without sodium bicarbonate, supplemented with 2\% FBS and Penicillin/Streptomycin, pH: 7.4) and incubated for $1 \mathrm{~h}$. Cells were then incubated at $37^{\circ} \mathrm{C}$ in a non-CO2 incubator that ready for experiments. Oligomycin $(10 \mu \mathrm{M}), \operatorname{FCCP}(2 \mu \mathrm{M})$, and rotenone/antimycin A $(5 \mu \mathrm{M})$ were prepared and placed into the sensor cartridge for the injection in the running procedure. The procedure of the assay was performed according to the guidelines from the XFe24 Seahorse Mitochondrial Respiration Mito Stress Test (Agilent technologies) [27]. For the evaluations of FAO percentage, etomoxir ( $40 \mu \mathrm{M})$ were added into the medium, $90 \mathrm{~min}$ before running the assay. FAO-dependent oxygen consumption was calculated as [OCR from groups without etomoxir - OCR from groups treated with etomoxir].

\section{Plasmids and transfection}

GFP-Sp1 and PTGES2 plasmids (HG19428-ACG, Sino Biological, Wayne, PA, USA) were transfected into cells with Poly-Jet ${ }^{\mathrm{TM}}$ Reagent (SignaGen Laboratories, Rockvillie, MD, USA) for overexpression. PTGS2 siRNA (Dharmacon, Lafayette, CO, USA) was transfected into cells with Lipofectamine RNAiMAX Reagent (Thermo Fisher Scientific) for knockdown. PLA2G5, ABHD8, and PTGS2 promoter were designed as a $1000 \mathrm{bp}$ sequence before the coding region. The information of the constructs was listed in Supplementary Table 4.

\section{Western blotting}

Protein samples were separated on SDS-PAGE and transferred onto the PVDF membrane (Bio-Rad). The PVDF membrane was blocked in $5 \%$ nonfat milk in TBST buffer at room temperature for an hour, and then incubated with specific primary antibodies (Supplementary Table 5) at $4^{\circ} \mathrm{C}$ overnight. After washing with TBST buffer, the membranes were incubated with the appropriate secondary antibodies for another one hour. Finally, the membranes were washed, and then developed by using T-Pro LumiLong Plus Chemiluminescent detection kit (T-Pro Biotechnology, New Taipei City, Taiwan).

\section{Real-time PCR}

The RNA sample was extracted by TRIzol (Thermo Fisher Scientific), and $1 \mu \mathrm{g}$ of total RNA was subjected to real-time PCR reagent using Prime Script ${ }^{T M}$ RT Reagent kit (Takara Bio. Inc, Shiga, Japan). The expression of each mRNA was determined using $2 \times$ SYBR real time master mix (AB Sciex) and the 
specific primers (Supplementary Table 6). GAPDH was used as the internal control. SYBR green fluorescence was then monitored using an ABI 7000 Sequence Detection System (AB Sciex).

\section{MitoPlates analysis for estimating the consumption of NADH/FADH-producing substrate}

MitoPlates S-1 (Biolog, Hayward, CA, USA) were used according to the manufacturer's instruction. To dissolve the substrates coated on MitoPlates, assay mixture containing saponin $(30 \mu \mathrm{g} / \mathrm{mL})$ was added into each well and the plate was incubated at $37^{\circ} \mathrm{C}$ for $1 \mathrm{~h}$. After the treatment with PGE2, cells were trypsinized and $1.5 \times 10^{6}$ cells were mixed with $1 \times$ Mitochondrial Assay Solution (MAS) and added equally into each well. The mixtures were incubated at $37^{\circ} \mathrm{C}$ for $2 \mathrm{~h}$ and the absorbance was detected at $590 \mathrm{~nm}$.

\section{Xenograft animal model}

TMZ-resistant U87MG-R ( $1 \times 10^{6}$ cells in $50 \mu$ L of DMEM) was injected into the back of 8-week-old CAnN.Cg-Foxn1nu/CrlBltw (BALB/c nude) male mice. Pt\#3-R cells $\left(1 \times 10^{6}\right.$ cells in $50 \mu \mathrm{L}$ of DMEM) were injected into the back of 8-week-old NOD.CB17-Prkdcscid/NcrCrl (NOD/SCID) male mice. A month after transplantation, mice were administrated with TMZ (10 mg/kg), ONO-8713 (15 mg/kg), and celecoxib (10 $\mathrm{mg} / \mathrm{kg}$ ) twice a week by intraperitoneal injection for another 8 weeks. After the treatment, the mice were then sacrificed and the tumors were excised by surgery to measure the weight and size. Tumor size was calculated according to the formula: $1 / 2$ * long side * (short side) ${ }^{2}$.

\section{Statistical analysis}

The data obtained were represented as means \pm S.E.M. Two-tailed unpaired Student's t-test or two-way ANOVA (animal experiments) were used to analyse the differences between the control and experimental groups. ${ }^{\star} P<0.05, \star \star P<0.01$, and ${ }^{\star \star \star} P<0.001$ were considered significant in all comparisons.

\section{Results}

\section{Importance of Sp1 in phospholipid metabolism to generate AA in TMZ-resistant GBM}

Metabolic reprogramming is critical for cancer progression.[28] In this study, we attempted to elucidate whether Sp1 is involved in metabolic reprogramming during the process of TMZ resistance acquisition in GBM. By analysing the RNA-Seq-based transcriptome data, it was observed that Sp1 knockdown downregulated the expression of phospholipid metabolic-related genes, including phospholipase A2 group V (PLA2G5), abhydrolase domain containing 8 (ABHD8), prostaglandin-endoperoxide synthase 2 (PTGS2)/cyclooxygenase 2 (COX2), prostaglandin E synthase 2 (PTGES2), prostaglandin D synthase (PTGDS), and aldo-keto reductase family 1 member C3 (AKR1C3) (Fig. 1A). Further, Sp1 knockout resulted in a significant decrease in the levels of several AA-derived metabolites, including PGE2, PGD2, and PGF2a; whereas the levels of 5(S)-HETE and 12(R)-HETE were upregulated (Fig. 1B). Overall, these 
results suggested that Sp1-regulated AA metabolism participates in the acquisition of TMZ resistance in GBM via the COX pathway.

\section{Importance of Sp1-regulated AA metabolism in TMZ- resistance acquisition in GBM}

To elucidate the role of AA metabolism in TMZ-resistance acquisition, the effects of inhibitors that target PLA2, PTGS2/COX2, ALOX5, and ALOX12 on TMZ-resistant U87MG-R cells were evaluated. As shown in Fig. 2A, pyrrophenone (PLA2 inhibitor) and celecoxib (PTGS2 inhibitor) synergistically enhanced TMZinduced cell death, whereas neither zileuton (ALOX5 inhibitor) nor ML-355 (ALOX12 inhibitor) exhibited any therapeutic effects (Fig. 2B). These results support the idea that the regulation of AA metabolism by $\mathrm{Sp} 1$, leading to the synthesis of prostaglandin and not lipoxygenase, is critical for the acquisition of TMZ resistance in GBM.

Moreover, to investigate the clinical relevance of Sp1-regulated prostaglandin synthesis, we collected 14 pairs of GBM specimens (primary vs. recurrent) for analysis (Supplementary Table 1). RNA-Seq revealed that the enzymes related to AA generation and prostaglandin metabolism, including PLA2G5, ABHD8, PTGS2, PTGES2, PTGDS, and AKR1C3, were obviously upregulated in the recurrent GBM specimens (Fig. 2C). Additionally, chromatin immunoprecipitation (ChIP)-Seq and reporter assay analyses revealed that Sp1 significantly enhanced the transcriptional activities of PLA2G5, ABHD8, PTGS2, PTGES2, PTGDS, and AKR1C3 with respect to prostaglandin synthesis in TMZ-resistant GBM cells by binding to their promoter regions (Fig. 2D-E). Overall, our results indicated the Sp1-regulated metabolic pathway from AA to prostaglandins is required for TMZ resistance acquisition in GBM.

\section{Enhancement of PGE2 synthesis in recurrent/resistant GBM}

Further, targeted metabolome analysis focusing on $\omega-3 / 6$ fatty acid metabolism was performed to determine the differences in the amounts of AA-related metabolites between paired (primary vs. recurrent) tissues from patients with GBM (Supplementary Table 2). As shown in Fig. 3A and Supplementary Fig. 1A, four out of a total five recurrent specimens exhibited significantly higher PGE2 and PGD2 levels. Additionally, we also estimated the amounts of AA-related metabolites via targeted arachidonate metabolome analysis. Thus, it was observed that the levels of PGD2 and PGE2 were also obviously higher in the recurrent tissue specimens than in normal brain tissue and primary specimens, suggesting that these two prostanoids are involved in TMZ resistance and tumour recurrence (Supplementary Fig. 1B). Further, as shown in Fig. 3B and Supplementary Fig. 1C, PGE2 significantly attenuated TMZinduced cytotoxicity in patient-derived GBM cells, Pt\#3, and U87MG. However, PGD2 did not affect cellular response to $\mathrm{TMZ}$.

Besides, compared with the wild type glioblastoma cell line, an increase in PGE2 secretion was observed in TMZ-resistant glioblastoma cell lines (Pt\#3-R, P1S, and U87MG-R cells), while a decrease was observed in U87MG-Sp1 cKO cells (Fig. 3C-D). Specifically, P1S cells, which exhibit TMZ resistance, secreted more PGE2 than patient-derived glioblastoma cells Pt\#3 (Fig. 3C, right). Further, long term TMZ 
treatment resulted in the enhancement of PTGS2 expression and PGE2 secretion (Fig. 3E and F and Supplementary Fig. 2A and 2B). It was also observed that GBM cells, Pt\#3 and U87MG, gradually showed increased tolerance to TMZ in the long-term treatment, and this increased tolerance was compromised by the PTGS2 inhibitor, celecoxib, resulting in a decrease in the number of adhered cells, an increase in the number of round-up cells, and the destruction of cell morphology (Supplementary Fig. 2C and 2D). These results supported the idea that the synthesis and secretion of PGE2 are significantly increased and required for the establishment of the recurrent and TMZ resistant GBM.

\section{Induction of mitochondrial fusion to rescue of TMZ- impaired respiration by PGE2 via EP1/EP3 cascades}

Given that mitochondrial dynamics act as regulators in cancer processes,[29-31] and that mitochondria play a critical role in the generation of bioenergy for $\mathrm{GBM},[17,18]$ we evaluated whether mitochondrial fission and fusion are involved in drug resistance in GBM. Thus, RNA-Seq showed that mitochondrial fusion-related proteins, such as MFN1, MFN2, and OPA1, were significantly upregulated in resistant Pt\#3$\mathrm{R}$ and recurrent GBM specimens (Fig. 4A), while mitochondrial fission-related proteins showed a different pattern (Supplementary Fig. 3A). Further, TEM also showed condensed mitochondria, which favours mitochondrial fusion, in TMZ-resistant GBM cell lines (Supplementary Fig. 3B). Moreover, consistent with PGE2 upregulation in long term TMZ-treated GBM cells, MFN1 and OPA1 levels showed a gradual increase (Fig. 4B). These results suggested that mitochondrial fusion, which enhances ATP production and respiration,[29-31] is upregulated in TMZ-resistant GBM cells. Further, Seahorse Mitochondrial Respiration Mito Stress Test revealed obviously enhanced mitochondrial activity in TMZ-resistant U87MG-R cells (Fig. 4C), and most importantly, PGE2 showed the ability to enhance ATP synthesis and mitochondrial activity in U87MG and Pt\#3 cells (Fig. 4D and Supplementary Fig. 3C). Additionally, mitochondrial fusion was observed in the cells incubated with PGE2 (Fig. 4E), suggesting that PGE2 enhanced ATP production by inducing mitochondrial fusion. Our results also revealed that PGE2 significantly attenuated TMZ-impaired mitochondrial respiration (Fig. 4F and Supplementary Fig. 3D). Besides, Sp1 knockout suppressed mitochondrial respiration, while PGE2 significantly rescued the diminished mitochondrial activity (Supplementary Fig. 3E). To identify the receptor responsible for PGE2regulated mitochondrial activity, the effects of EP1-EP4 receptor antagonists (ONO-8713: EP1, PF04418948: EP2, ONO-AE-240: EP3, and ONO-AE3-208: EP4) were investigated. Thus, it was observed that ONO-8713 and ONO-AE3-240, which inhibit EP1 and EP3 receptors, respectively, effectively attenuated the enhancement of ATP production by PGE2 (Fig. 4G and Supplementary Fig. 3F). Therefore, our results indicated that to establish TMZ resistance in GBM, Sp1-regulated PGE2 synthesis promoted mitochondrial fusion so as to enhance ATP production and rescue TMZ-impaired mitochondrial respiration through EP1 and EP3 cascades.

\section{PGE2-based promotion of FAO and TCA cycle progression contribute to increased ATP production}


In this study, we also investigated the contribution of the key proteins and metabolites that are regulated by Sp1 and PGE2 to the upregulation of ATP production after mitochondrial fusion.[29-31] In this regard, RNA-Seq revealed that Sp1 knockdown downregulated the expression of several mitochondrial ATP production-related genes, which were obviously upregulated in recurrent specimens (Table 1, Fig. 5A, and Supplementary Fig. 4A). Among these genes, CPT1A and ACAA2, which play predominant roles in the regulation of FAO pathways, were consistently and significantly upregulated in the recurrent $G B M$ and TMZ-resistant GBM specimens (Fig. 5A and B, and Supplementary Fig. 4B). These results are consistent with our hypothesis that Sp1 enhances lipid metabolism in TMZ-resistant GBM, with CPT1A as the ratelimiting step of $\mathrm{FAO},[32]$ facilitating the transfer of fatty acids into the mitochondria for bioenergy generation. Therefore, we confirmed that PGE2 significantly enhances the protein expression of CPT1A without affecting the levels of glycolysis-related proteins, LDHA, and NQO1 (Fig. 5C, and Supplementary Fig. 4C). Moreover, PGE2 significantly increased the percentage contribution of FAO to mitochondrial respiration. This is an important metabolic characteristic of TMZ-resistant GBM (Fig. 5D and Supplementary Fig. 4D). These results indicated that Sp1-regulated PGE2 enhances FAO by inducing CPT1A expression to acquire TMZ resistance.

Supporting the importance of FAO in drug resistance, untargeted metabolome analysis showed a significant increase in the levels of FAO-related metabolites, including acyl-carnitine, trans-2-Enoyl-CoA, 3hydroxyacyl-CoA, and acetyl-CoA, in TMZ-resistant GBM cells (Fig. 5E). Consistent with this observation, for TCA cycle progression, PGE2 significantly increased the levels of citrate, cis-aconitate, a-ketoglutarate, succinate, and malate (Fig. 5F). Furthermore, to generate bioenergy, PGE2 enhanced the consumption of fatty acid- and TCA cycle-related substrates, such as short chain (Acetyl-L-carnitine) and long chain (Pamitoyl-L-carnitine) fatty acids, citric acid, cis-aconitic acid, fumaric acid, and L-malic acid, (Fig. 5G), implying that in the presence of PGE2, GBM cells show preference for the consumption of fatty acids as a major source of bioenergy. Therefore, Sp1-regulated PGE2 increases the number of metabolites in FAO and drives TCA cycle progression after mitochondrial fusion to rescue TMZ-induced nutrient deprivation and mitochondria damage.

\section{Blockade of PGE2 action by EP1 and EP3 antagonists suppresses TMZ-resistant GBM}

To prevent GBM from acquiring PGE2-based TMZ resistance, several compounds targeting PGE2 synthesis (celecoxib) and receptors (ONO-8713, PF-04418948, ONO-AE3-240, ONO-AE3-208) were evaluated. Among the candidate PGE2 receptor-targeting compounds, the EP1 antagonist, ONO-8713, and the EP3 antagonist, ONO-AE3-240, significantly abolished PGE2-induced resistance (Fig. 6A and B and Supplementary Fig. 5A and S5B). Additionally, ONO-8713 and ONO-AE3-240 significantly inhibited the survival of multiple GBM cells with or without TMZ resistance, including T98G cells expressing MGMT (Fig. 6C and Supplementary Fig. 5C). Further, we evaluated the therapeutic effect of ONO-8713, which showed the strongest potential in suppressing resistant GBM tumour growth based on experiments involving a mouse xenograft brain tumour model. As shown in Fig. 6D, both ONO-8713 and celecoxib 
significantly inhibited Pt\#3-R tumour growth. Additionally, ON0-8713 also significantly inhibited U87MGR-derived tumour growth (Fig. 6E).

\section{Discussion}

Given that GBM utilizes mitochondrial oxidation to generate bioenergy, $[17,18]$ we investigated which part of the ATP-generating system is upregulated in TMZ-resistant GBM. Our study indicated that FAO and TCA cycles are upregulated in TMZ-resistant GBM owing to the action of PGE2. It has also been reported that FAO plays an important role in tumour development in nutrient-deprived environment[18] and cancer metastasis. $[19,20]$ Particularly, increases fatty acid uptake and the alteration of lipid metabolism are associated with drug resistance in cancers and provide the essential bioenergy for cancer survival based on the enhancement of FAO efficacy.[33] For example, the resistance of cancers to antiangiogenic drugs is due to the alteration of FAO and lipid metabolism in the tumour environment.[34] Therefore, therapeutic strategies that target the alteration of lipid metabolism, such as blocking cancer-associated adipocytemediated fatty acid production followed by the inhibition of free fatty acid uptake by cancer cells, have been designed to combat drug resistance.[34] However, strategies that target FAO for the treatment of drug resistant cancer remain limited. Even though etomoxir reportedly blocks FAO by inhibiting CPT1 and suppressing GBM cell growth,[18] our experimental results revealed that etomoxir is not effective in inhibiting drug resistance in GBM (data not shown). Hence, the PGE2/EP1 cascade, which has a significant regulatory effect on CPT1-dependent FAO, is expected to be a potential target for overcoming drug resistance.

Celecoxib is a well-known COX2/PTGS2 inhibitor that attenuates the synthesis of PGE2, and reportedly, kills GBM cells.[35] However, it failed a phase II clinical trial owing to its poor permeability with respect to crossing the blood-brain barrier.[36, 37] Therefore, a treatment strategy that targets the PGE2-induced functional pathway, such as PGE2 receptor (EP1-EP4) blocking, instead of COX2/PTGS2-mediated PGE2 synthesis suppression, is an alternative medical option. Reportedly, PGE2 receptors are associated with cancer malignancy, while their antagonists have also shown tumorigenesis inhibition effects.[38] Among EP1-EP4 receptors, EP1 is involved in the activation of cancer cell migration and invasion,[39-41] supports tumour adaptation to hypoxia,[42] and enhances cancer initiation.[43, 44] EP2 is known to be involved in the induction of angiogenesis[38] and in the suppression of antitumor immune response.[45] Further, its inhibition in glioma results in tumour growth suppression.[46] Conversely, the role of EP3 in tumorigenesis is still controversial.[38] The results of our previous study showed that the EP3 antagonist, ONO-AE3-240, also exerts cell cytotoxicity on TMZ-resistant GBM cells. Additionally, it has been demonstrated that EP4 is involved in cancer migration, metastasis, and aberrant DNA methylation.[4749] The results of this study indicated that the EP1 antagonist, ONO-8713, is capable of blocking PGE2induced mitochondrial activation and suppressing the survival of TMZ-resistant GBM cells. Therefore, EP antagonists, instead of celecoxib, have potential to attenuate drug resistance by blocking PGE2-mediated signalling in GBM. Overall, PGE2 induces TMZ resistance in GBM via mitochondria-mediated FAO activation under the control of EP1 and EP3. Further combining TMZ with an EP1 antagonist present as a potential combination therapeutic strategy for TMZ-resistant GBM. Therefore, the development of EP1 
antagonists that can cross the blood-brain barrier during the treatment of TMZ-resistant GBM have great application prospects.

\section{Conclusion}

Sp1 increases PGE2 synthesis through enhancing gene expression involved in AA metabolism to PGE2 in recurrent GBM, leading to TMZ resistance. Further, PGE2 increases mitochondrial fusion, resulted in the enhancement of FAO and TCA cycle to increase the ATP production, through the EP1 and EP3 receptors. Moreover, EP1 antagonist ONO-8713 exhibits a potentially therapeutic effect on TMZ-resistant GBM in vivo and in vitro (Figure 6f).

\section{Abbreviations}

AA

arachidonic acid

$A B C$

ATP binding cassette

ABHD

abhydrolase domain containing

ACAA

acetyl-coenzyme $A$ acyltransferase

AKR1C3

aldo-keto reductase family 1 member $\mathrm{C} 3$

ALOX

arachidonate lipoxygenase

CAMP

cyclic adenosine $3^{\prime}, 5^{\prime}$-cyclic monophosphates

cox2

cyclooxygenase-2

ChIP

Chromatin immunoprecipitation

CPT

carnitine palmitoyltransferase

CYP

cytochrome $\mathrm{p} 450$

ENO2

enolase 2

EP

E-type prostanoid receptor

FAO 
fatty acid $\beta$-oxidation

GBM

glioblastoma

GFA

glial fibrillary acidic protein

HETE

hydroxyeicosatetraenoic

IDH-1

Isocitrate dehydrogenase 1

LDHA

lactate dehydrogenase $\mathrm{A}$

MFN1/2

mitofusin $1 / 2$

MGMT

06-methylguanine-DNA methyltransferase

OPA1

optic Atrophy 1

PDK1

pyruvate dehydrogenase kinase-1

PG

Prostaglandin

PLA2G5

phospholipase A2 group $V$

PTGES2

prostaglandin E synthase 2

PTGS2

prostaglandin-endoperoxide synthase 2

Sp1

Specificity protein 1

TCA

tricarboxylic acid

TEM

Transmission electron microscopy

TMZ

Temozolomide

\section{Declarations}

Ethics approval and consent to participate 
The use of human specimens adhered to the institutional human ethics guidelines and was approved by the Clinical Research Ethics Committee at Taipei Medical University Hospital (No. 201006011 and No. 201402018) and Chang Gung Memorial Hospital (No. 201901848B0). Animal experiments were conducted according to the guideline and approved by the Institutional Animal Care and Use Committee of Taipei Medical University (No. LAC-2018-0401).

\section{Consent for publication}

Not applicable

\section{Availability of data and materials}

The author declared that all and the other data supporting the findings of this study are available within the paper. The raw data that support the findings of this study are available from the corresponding author upon reasonable request.

\section{Competing interests}

The authors declare no competing interests.

\section{Funding}

Ministry of Science and Technology of Taiwan: MOST108-2320-B-038-012, MOST108-2628-B-038-005, MOST109-2320-B-038-065, MOST109-2628-B-038-017-, MOST109-2320-B-182A-005-MY3 and MOST1102628-B-038-021-; Taipei Medical University-Shuang Ho Hospital: 110TMU-SHH-16; Chang Gung Memorial Hospital: CMRPG2K0251

\section{Author contributions}

Conceptualization: Y.T. Tsai, W.L. Lo, W.C. Chang, T.I. Hsu

Methodology: Y.T. Tsai, W.L. Lo, T.I. Hsu

Investigation: Y.T. Tsai, P.Y. Chen, J.Y. Chuang, C.Y.J. Wu, W.B. Yang, K.Y. Chang, C.Y. Hung, T.J. Kao, T.I. Hsu

Visualization: Y.T. Tsai, W.B. Yang

Supervision: W.C. Chang, T.I. Hsu

Writing-original draft: Y.T. Tsai, W.L. Lo

Writing-review \& editing: U. Kikkawa, W.C. Chang, T.I. Hsu

\section{Acknowledgments}


We thank the support by TMU Research Center of Cancer Translational Medicine from The Featured Areas Research Center Program within the framework of the Higher Education Sprout Project by the Ministry of Education in Taiwan. We are grateful to Ono Pharmaceutical Co., Ltd. for providing EP1, EP3, and EP4 antagonists.

\section{References}

1. Hanif F, Muzaffar K, Perveen K, Malhi SM, Simjee Sh U. Glioblastoma Multiforme: A Review of its Epidemiology and Pathogenesis through Clinical Presentation and Treatment. Asian Pac J Cancer Prev. 2017;18:3-9.

2. Chang KY, Hsu TI, Hsu CC, Tsai SY, Liu JJ, Chou SW, Liu MS, Liou JP, Ko CY, Chen KY, et al. Specificity protein 1-modulated superoxide dismutase 2 enhances temozolomide resistance in glioblastoma, which is independent of O(6)-methylguanine-DNA methyltransferase. Redox Biol. 2017;13:655-64.

3. Chang KY, Huang CT, Hsu TI, Hsu CC, Liu JJ, Chuang CK, Hung JJ, Chang WC, Tsai KK, Chuang JY. Stress stimuli induce cancer-stemness gene expression via Sp1 activation leading to therapeutic resistance in glioblastoma. Biochem Biophys Res Commun. 2017;493:14-9.

4. Chuang JY, Lo WL, Ko CY, Chou SY, Chen RM, Chang KY, Hung JJ, Su WC, Chang WC, Hsu TI. Upregulation of CYP17A1 by Sp1-mediated DNA demethylation confers temozolomide resistance through DHEA-mediated protection in glioma. Oncogenesis. 2017;6:e339.

5. Yang WB, Chuang JY, Ko CY, Chang WC, Hsu TI. Dehydroepiandrosterone Induces Temozolomide Resistance Through Modulating Phosphorylation and Acetylation of Sp1 in Glioblastoma. Mol Neurobiol. 2019;56:2301-13.

6. Lo WL, Hsu TI, Yang WB, Kao TJ, Wu MH, Huang YN, Yeh SH, Chuang JY: Betulinic Acid-Mediated Tuning of PERK/CHOP Signaling by Sp1 Inhibition as a Novel Therapeutic Strategy for Glioblastoma. Cancers (Basel) 2020, 12.

7. Yang WB, Hsu CC, Hsu TI, Liou JP, Chang KY, Chen PY, Liu JJ, Yang ST, Wang JY, Yeh SH, et al. Increased activation of HDAC1/2/6 and Sp1 underlies therapeutic resistance and tumor growth in glioblastoma. Neuro Oncol. 2020;22:1439-51.

8. Tsai YT, Wu CC, Ko CY, Hsu TI, Chang WC, Lo WL, Chuang JY. Correlation between the expression of cancer stem cell marker BMI1 and glioma prognosis. Biochem Biophys Res Commun. 2021;550:113-9.

9. Lin HY, Ko CY, Kao TJ, Yang WB, Tsai YT, Chuang JY, Hu SL, Yang PY, Lo WL, Hsu TI: CYP17A1 Maintains the Survival of Glioblastomas by Regulating SAR1-Mediated Endoplasmic Reticulum Health and Redox Homeostasis. Cancers (Basel) 2019, 11.

10. Chen TC, Chuang JY, Ko CY, Kao TJ, Yang PY, Yu CH, Liu MS, Hu SL, Tsai YT, Chan H, et al. AR ubiquitination induced by the curcumin analog suppresses growth of temozolomide-resistant glioblastoma through disrupting GPX4-Mediated redox homeostasis. Redox Biol. 2020;30:101413.

11. Wang D, Dubois RN. Eicosanoids and cancer. Nat Rev Cancer. 2010;10:181-93. 
12. Wang D, Dubois RN. Cyclooxygenase-2: a potential target in breast cancer. Semin Oncol. 2004;31:64-73.

13. Hambek M, Baghi M, Wagenblast J, Schmitt J, Baumann H, Knecht R. Inverse correlation between serum PGE2 and T classification in head and neck cancer. Head Neck. 2007;29:244-8.

14. Tian Y, Yang T, Yu S, Liu C, He M, Hu C. Prostaglandin E2 increases migration and proliferation of human glioblastoma cells by activating transient receptor potential melastatin 7 channels. J Cell Mol Med. 2018;22:6327-37.

15. Cook PJ, Thomas R, Kingsley PJ, Shimizu F, Montrose DC, Marnett LJ, Tabar VS, Dannenberg AJ, Benezra R. Cox-2-derived PGE2 induces Id1-dependent radiation resistance and self-renewal in experimental glioblastoma. Neuro Oncol. 2016;18:1379-89.

16. Liberti MV, Locasale JW. The Warburg Effect: How Does it Benefit Cancer Cells? Trends Biochem Sci. 2016;41:211-8.

17. Mounier C, Bouraoui L, Rassart E. Lipogenesis in cancer progression (review). Int J Oncol. 2014;45:485-92.

18. Kant S, Kesarwani P, Prabhu A, Graham SF, Buelow KL, Nakano I, Chinnaiyan P. Enhanced fatty acid oxidation provides glioblastoma cells metabolic plasticity to accommodate to its dynamic nutrient microenvironment. Cell Death Dis. 2020;11:253.

19. van Weverwijk A, Koundouros $N$, Iravani $M$, Ashenden $M, G a o$ Q, Poulogiannis $G$, Jungwirth $U$, Isacke CM. Metabolic adaptability in metastatic breast cancer by AKR1B10-dependent balancing of glycolysis and fatty acid oxidation. Nat Commun. 2019;10:2698.

20. Rozeveld CN, Johnson KM, Zhang L, Razidlo GL. KRAS Controls Pancreatic Cancer Cell Lipid Metabolism and Invasive Potential through the Lipase HSL. Cancer Res. 2020;80:4932-45.

21. Louis DN, Perry A, Reifenberger G, von Deimling A, Figarella-Branger D, Cavenee WK, Ohgaki $H$, Wiestler OD, Kleihues P, Ellison DW. The 2016 World Health Organization Classification of Tumors of the Central Nervous System: a summary. Acta Neuropathol. 2016;131:803-20.

22. Komori T. The 2016 WHO Classification of Tumours of the Central Nervous System: The Major Points of Revision. Neurol Med Chir (Tokyo). 2017;57:301-11.

23. Aquilanti E, Miller J, Santagata S, Cahill DP, Brastianos PK. Updates in prognostic markers for gliomas. Neuro Oncol. 2018;20:vii17-26.

24. Jung CS, Foerch C, Schanzer A, Heck A, Plate KH, Seifert V, Steinmetz H, Raabe A, Sitzer M. Serum GFAP is a diagnostic marker for glioblastoma multiforme. Brain. 2007;130:3336-41.

25. Calvo SE, Clauser KR, Mootha VK. MitoCarta2.0: an updated inventory of mammalian mitochondrial proteins. Nucleic Acids Res. 2016;44:D1251-7.

26. Tsai YT, Wu AC, Yang WB, Kao TJ, Chuang JY, Chang WC, Hsu TI. ANGPTL4 Induces TMZ Resistance of Glioblastoma by Promoting Cancer Stemness Enrichment via the EGFR/AKT/4E-BP1 Cascade. Int J Mol Sci 2019, 20. 
27. Divakaruni AS, Paradyse A, Ferrick DA, Murphy AN, Jastroch M. Analysis and interpretation of microplate-based oxygen consumption and pH data. Methods Enzymol. 2014;547:309-54.

28. Faubert B, Solmonson A, DeBerardinis RJ. Metabolic reprogramming and cancer progression. Science 2020, 368.

29. Simula L, Nazio F, Campello S. The mitochondrial dynamics in cancer and immune-surveillance. Semin Cancer Biol. 2017;47:29-42.

30. Trotta AP, Chipuk JE. Mitochondrial dynamics as regulators of cancer biology. Cell Mol Life Sci. 2017;74:1999-2017.

31. Bordi M, Nazio F, Campello S. The Close Interconnection between Mitochondrial Dynamics and Mitophagy in Cancer. Front Oncol. 2017;7:81.

32. Schlaepfer IR, Joshi M. CPT1A-mediated Fat Oxidation, Mechanisms, and Therapeutic Potential. Endocrinology 2020, 161.

33. Germain N, Dhayer M, Boileau M, Fovez Q, Kluza J, Marchetti P. Lipid Metabolism and Resistance to Anticancer Treatment. Biology (Basel) 2020, 9.

34. Cao Y. Adipocyte and lipid metabolism in cancer drug resistance. J Clin Invest. 2019;129:3006-17.

35. Kang KB, Zhu C, Yong SK, Gao Q, Wong MC. Enhanced sensitivity of celecoxib in human glioblastoma cells: Induction of DNA damage leading to p53-dependent G1 cell cycle arrest and autophagy. Mol Cancer. 2009;8:66.

36. Penas-Prado M, Hess KR, Fisch MJ, Lagrone LW, Groves MD, Levin VA, De Groot JF, Puduvalli VK, Colman H, Volas-Redd G, et al. Randomized phase II adjuvant factorial study of dose-dense temozolomide alone and in combination with isotretinoin, celecoxib, and/or thalidomide for glioblastoma. Neuro Oncol. 2015;17:266-73.

37. Novakova I, Subileau EA, Toegel S, Gruber D, Lachmann B, Urban E, Chesne C, Noe CR, Neuhaus W. Transport rankings of non-steroidal antiinflammatory drugs across blood-brain barrier in vitro models. PLoS One. 2014;9:e86806.

38. O'Callaghan G, Houston A. Prostaglandin E2 and the EP receptors in malignancy: possible therapeutic targets? Br J Pharmacol. 2015;172:5239-50.

39. Yang SF, Chen MK, Hsieh YS, Chung TT, Hsieh YH, Lin CW, Su JL, Tsai MH, Tang CH. Prostaglandin E2/EP1 signaling pathway enhances intercellular adhesion molecule 1 (ICAM-1) expression and cell motility in oral cancer cells. J Biol Chem. 2010;285:29808-16.

40. Kim SH, Park YY, Kim SW, Lee JS, Wang D, DuBois RN. ANGPTL4 induction by prostaglandin E2 under hypoxic conditions promotes colorectal cancer progression. Cancer Res. 2011;71:7010-20.

41. Zhang Y, Liu Q, Zhang M, Yu Y, Liu X, Cao X. Fas signal promotes lung cancer growth by recruiting myeloid-derived suppressor cells via cancer cell-derived PGE2. J Immunol. 2009;182:3801-8.

42. Kaidi A, Qualtrough D, Williams AC, Paraskeva C. Direct transcriptional up-regulation of cyclooxygenase-2 by hypoxia-inducible factor (HIF)-1 promotes colorectal tumor cell survival and enhances HIF-1 transcriptional activity during hypoxia. Cancer Res. 2006;66:6683-91. 
43. Watanabe K, Kawamori T, Nakatsugi S, Ohta T, Ohuchida S, Yamamoto H, Maruyama T, Kondo K, Narumiya S, Sugimura T, Wakabayashi K. Inhibitory effect of a prostaglandin E receptor subtype $\mathrm{EP}(1)$ selective antagonist, ONO-8713, on development of azoxymethane-induced aberrant crypt foci in mice. Cancer Lett. 2000;156:57-61.

44. O'Callaghan G, Ryan A, Neary P, O'Mahony C, Shanahan F, Houston A. Targeting the EP1 receptor reduces Fas ligand expression and increases the antitumor immune response in an in vivo model of colon cancer. Int J Cancer. 2013;133:825-34.

45. Kalinski P. Regulation of immune responses by prostaglandin E2. J Immunol. 2012;188:21-8.

46. Qiu J, Li Q, Bell KA, Yao X, Du Y, Zhang E, Yu JJ, Yu Y, Shi Z, Jiang J. Small-molecule inhibition of prostaglandin $\mathrm{E}$ receptor 2 impairs cyclooxygenase-associated malignant glioma growth. $\mathrm{Br} \mathrm{J}$ Pharmacol. 2019;176:1680-99.

47. Buchanan FG, Gorden DL, Matta P, Shi Q, Matrisian LM, DuBois RN. Role of beta-arrestin 1 in the metastatic progression of colorectal cancer. Proc Natl Acad Sci U S A. 2006;103:1492-7.

48. Xia S, Ma J, Bai X, Zhang H, Cheng S, Zhang M, Zhang L, Du M, Wang Y, Li H, et al. Prostaglandin E2 promotes the cell growth and invasive ability of hepatocellular carcinoma cells by upregulating cMyc expression via EP4 receptor and the PKA signaling pathway. Oncol Rep. 2014;32:1521-30.

49. Xia D, Wang D, Kim SH, Katoh H, DuBois RN. Prostaglandin E2 promotes intestinal tumor growth via DNA methylation. Nat Med. 2012;18:224-6.

\section{Figures}




\section{Figure 1}

A

Phospholipid-AA metabolism related genes
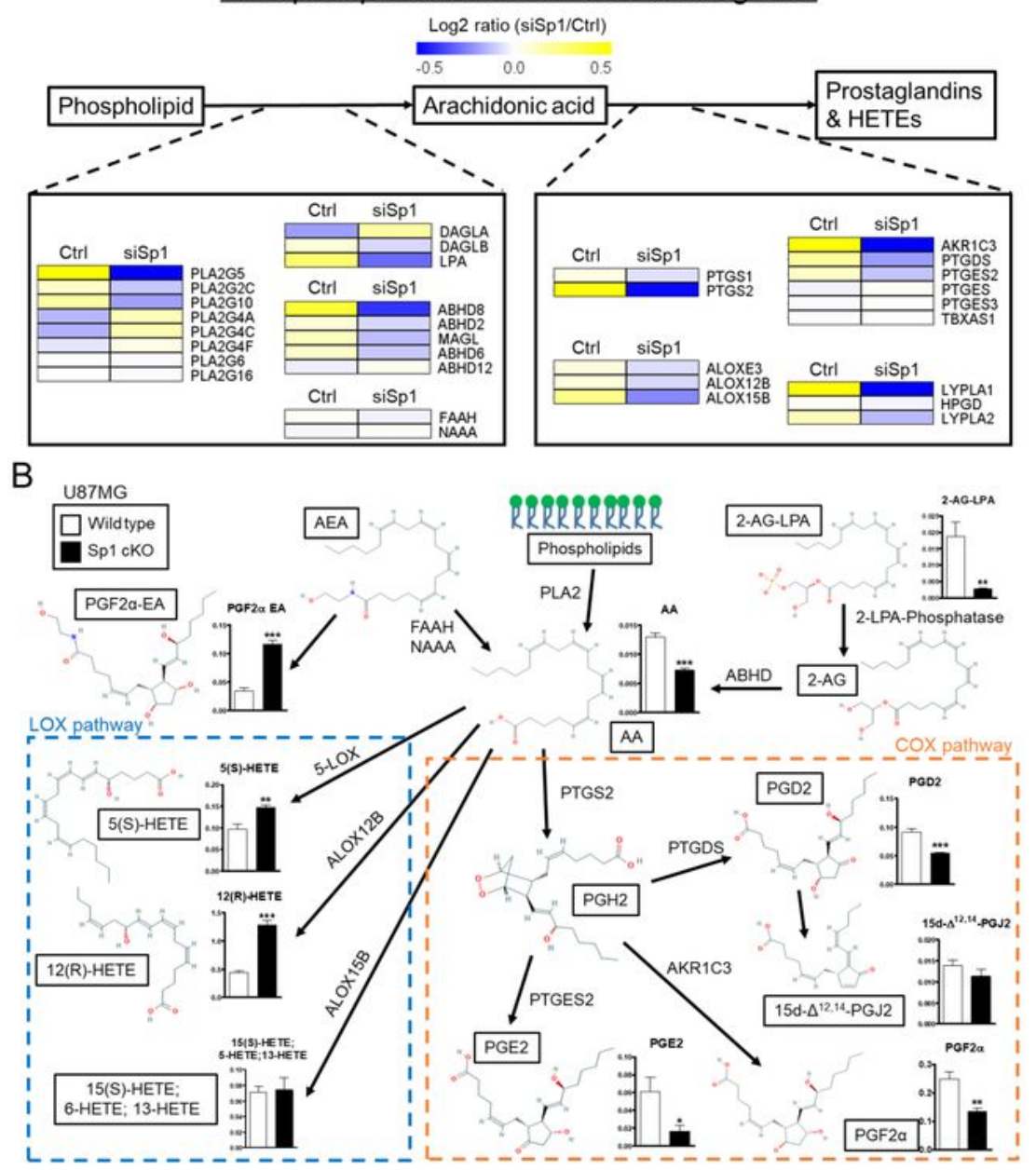

\section{Figure 1}

Regulation of phospholipid and AA metabolism by Sp1. (A) Sp1-regulated metabolic genes. After Sp1 knockdown by siRNA, U87MG cell RNA was collected and subjected to RNA-Seq. (B) AA-related metabolite alteration determined via UHPLC-QTOF-MS. U87MG and U87MG-Sp1 cKO cells were subjected to mass spectrometry-based metabolomics analysis. The alteration of metabolite levels was illustrated by performing two-tailed unpaired Student's t test. The chemical structures were obtained from PubChem, 
National Library of Medicine. The complete definitions of the different abbreviations are provided in Table S5.

\section{Figure 2}

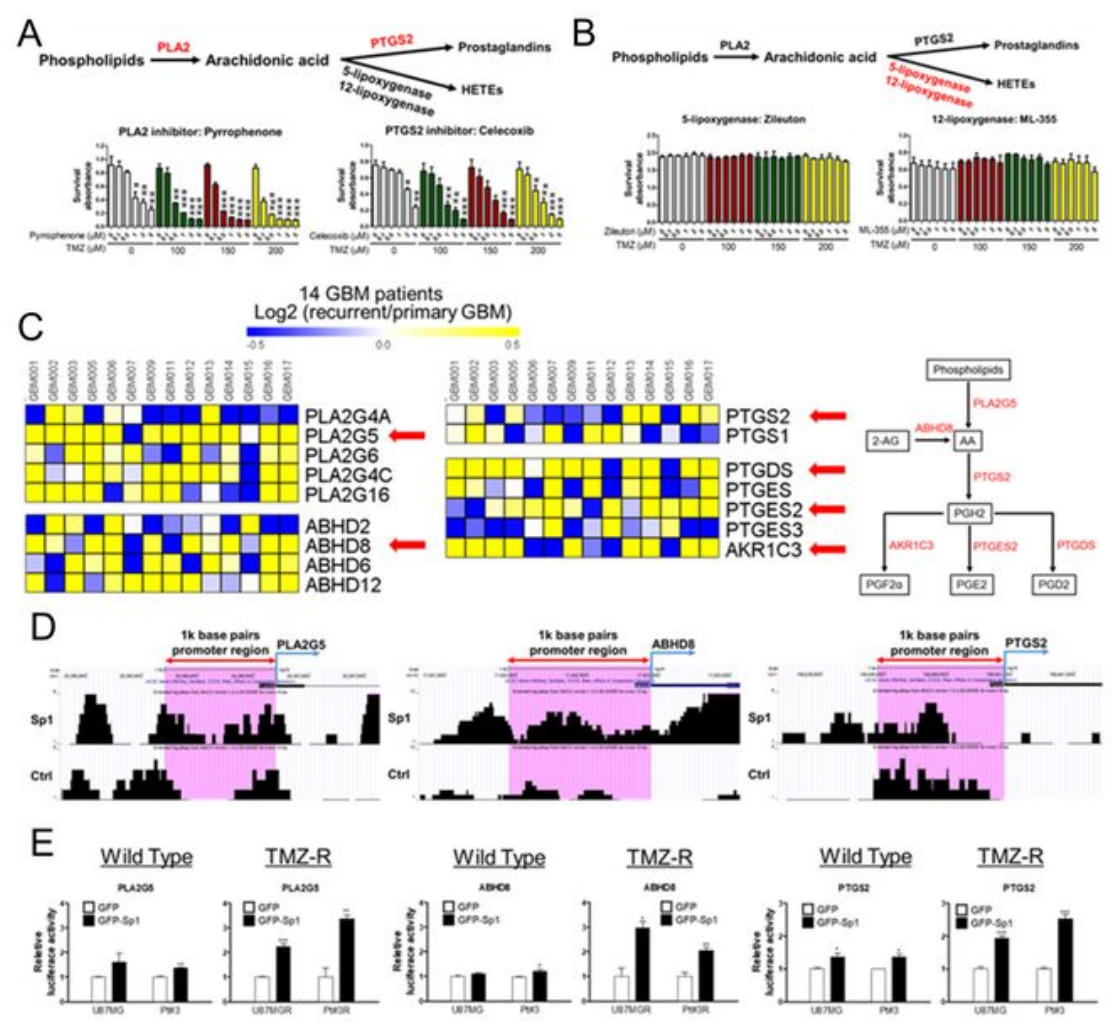

\section{Figure 2}

The Sp1-regulated COX2/PTGS pathway in recurrent glioblastoma. Effects of multiple inhibitors, including (A) Pyrrophenone (left) and celecoxib (right), (B) Zileuton (left) and ML-355 (right) on the viability of U87MG-R cells. The cells were treated with TMZ in the presence of the indicated inhibitors for 
$72 \mathrm{~h}$, and cell viability was determined via MTT assay. (C) Paired primary and recurrent glioblastoma specimens. Samples were collected from 14 patients and subjected to RNA-Seq. The red arrow-marked genes play important roles in the metabolic pathway involving the synthesis of prostaglandins from $A A$ (the COX pathway) as shown on the right panel. (D) Defined promoter regions of PLA2G5, ABHD8, and PTGS2. The binding regions of Sp1 were determined via ChIP-Seq. (E) Sp1-induced activities of pGL2conjugated promoter constructs, including PLA2G5, ABHD8, and PTGS2. Luciferase reporter assay was employed to analyse the promoter activities in wild type and TMZ-resistant glioblastoma cells. Data were analysed by performing two-tailed unpaired Student's $t$ test. 


\section{Figure 3}

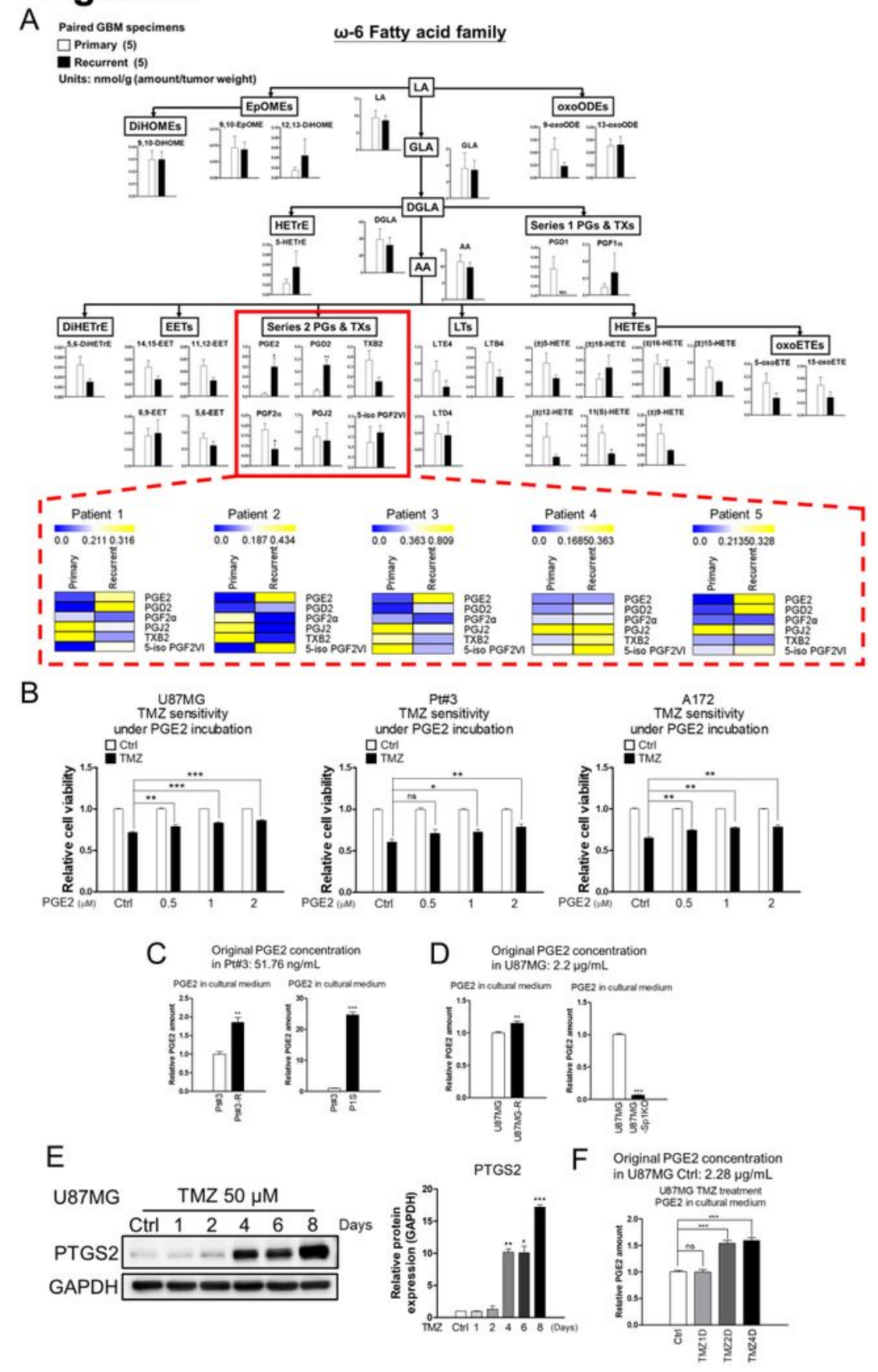

\section{Figure 3}

Effect of Sp1-regulated PGE2 production and secretion on TMZ-resistant glioblastoma. (A) Targeted $\omega-6$ fatty acid metabolome analysis for paired primary and recurrent glioblastoma specimens from five patients based on UHPLC-QTOF-MS. Data in bar plots were analysed by performing two-tailed unpaired Student's $t$ test. The alteration in prostanoids from each patient was expressed as a heatmap. The full definitions of the different abbreviations are provided in Table S6. (B) Effect of PGE2 on glioblastoma cell 
viability. After treatment with TMZ in the presence of PGE2 for four days, cell viability was estimated via MTT assay. (C), (D) Level of PGE2 in culture media estimated via ELISA. (E), (F) Cell lysates after treatment with TMZ for the indicated time interval analysed via Western blotting. The level of PGE2 in the culture media was determined via ELISA. Data and the quantifications were analysed by performing twotailed unpaired Student's t test.

\section{Figure 4}
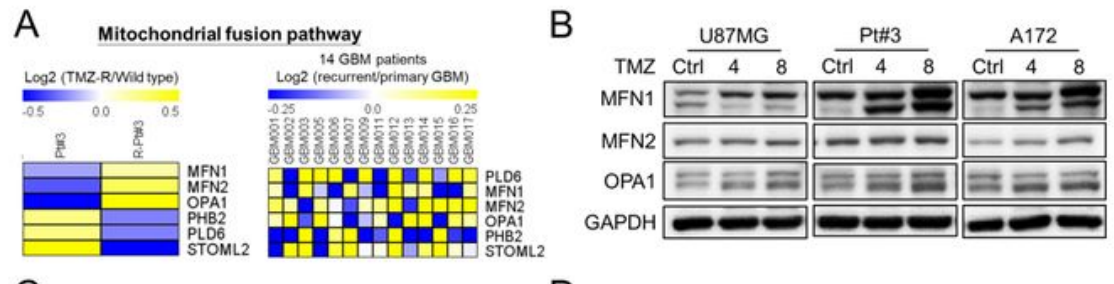

C
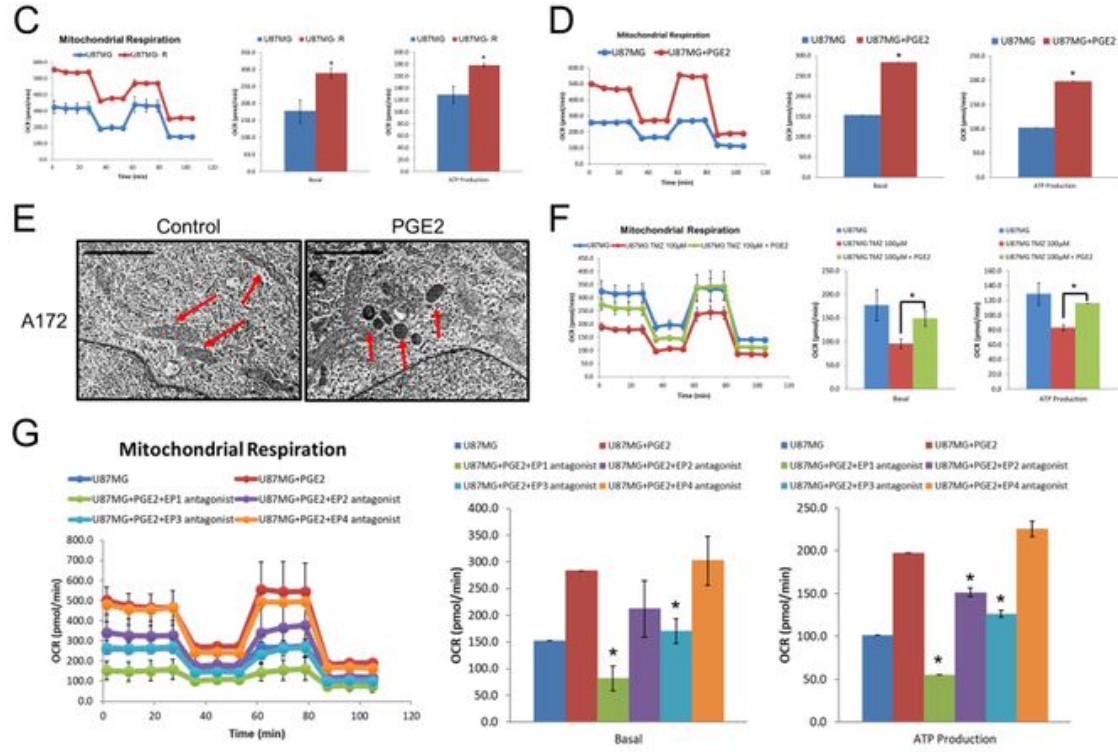

\section{Figure 4}


Effect of PGE2 on mitochondrial function. (A) RNA expression in cell line from patient with glioblastoma and paired glioblastoma specimens. (B) Effect of TMZ on mitochondrial fusion-related protein expression. (C) Mitochondrial activities of U87MG, U87MG-R, and U87MG-Sp1KO cells determined by performing Seahorse XF Mito Stress tests. (D) Mitochondrial activity determined via Seahorse XF Mito Stress tests after treatment with PGE2 for four days. (E) TEM images of cells. The scale bar was $2 \mu \mathrm{m}$. (F) Mitochondrial activity after treatment with TMZ in the presence or absence of PGE2 for four days. (G) Mitochondrial activity after treatment with PGE2 in the presence of each antagonist for four days. Statistical data were analysed by performing two-tailed unpaired Student's $t$ test. 


\section{Figure 5}
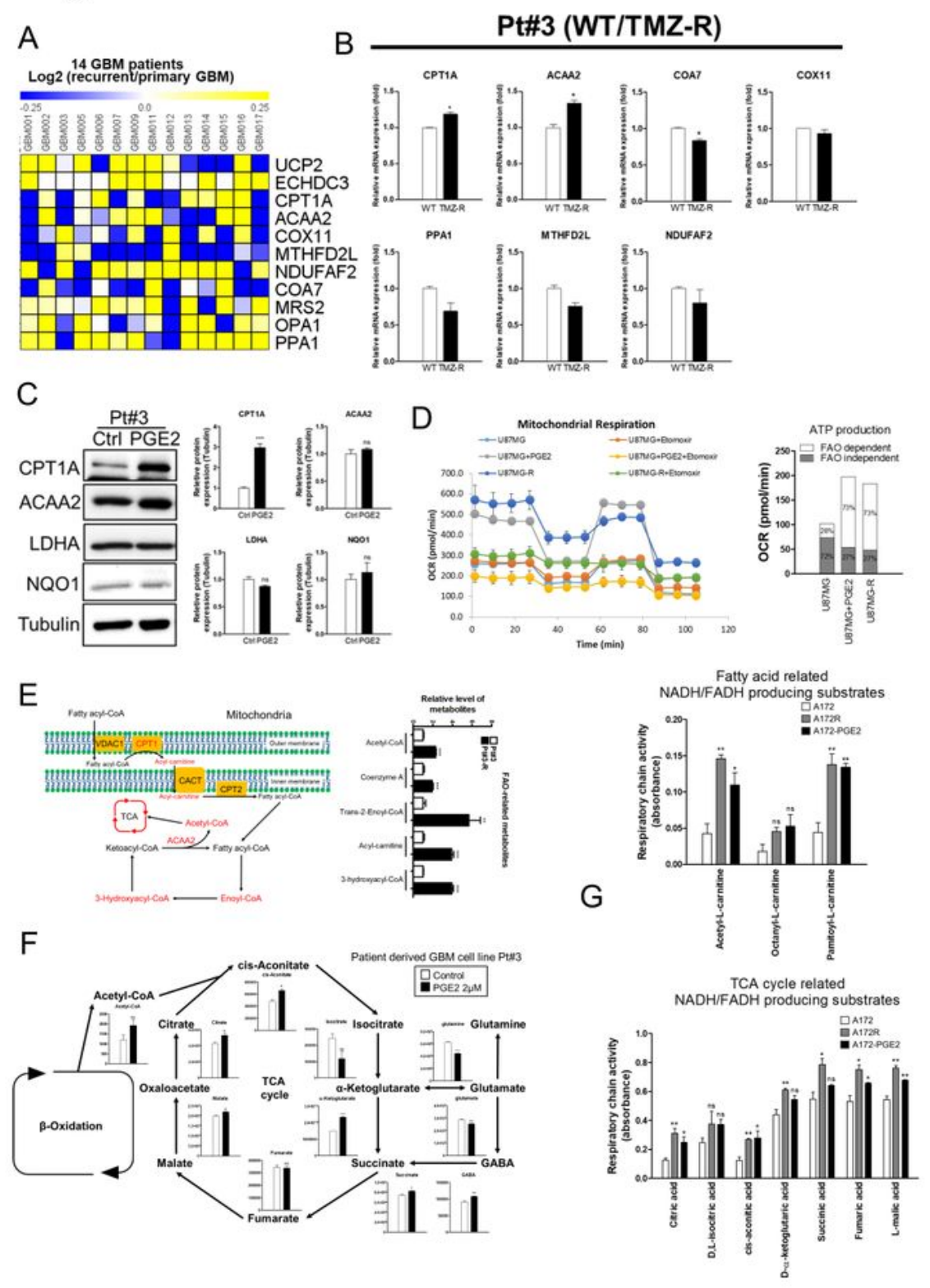

\section{Figure 5}

Effect of Sp1-regulated PGE2 on FAO and TCA cycles. (A) RNA expression in paired glioblastoma specimens. (B) Expression of mRNA in Pt\#3 and Pt\#3-R cells determined via real-time PCR. (C) Cell lysates after treatment with PGE2 for four days based on Western blotting. (D) Percentage of FAO measured by performing Seahorse XF Mito Stress Tests. (E) left panel: FAO metabolic pathway; right panel: wild type Pt\#3 and TMZ-resistant Pt\#3-R cells subjected to metabolomics analysis. (F) Pt\#3 cells 
after treatment with PGE2 for four days based on targeted metabolomics analysis. The alteration of the levels of the different metabolites is illustrated using bar plots. Data were analysed by performing twotailed unpaired Student's t test. (G) A172 and A172R cells after treatment with PGE2 for four days based on MitoPlate assay for $2 \mathrm{~h}$. Data were analysed by performing two-tailed unpaired Student's t test.

\section{Figure 6}

A
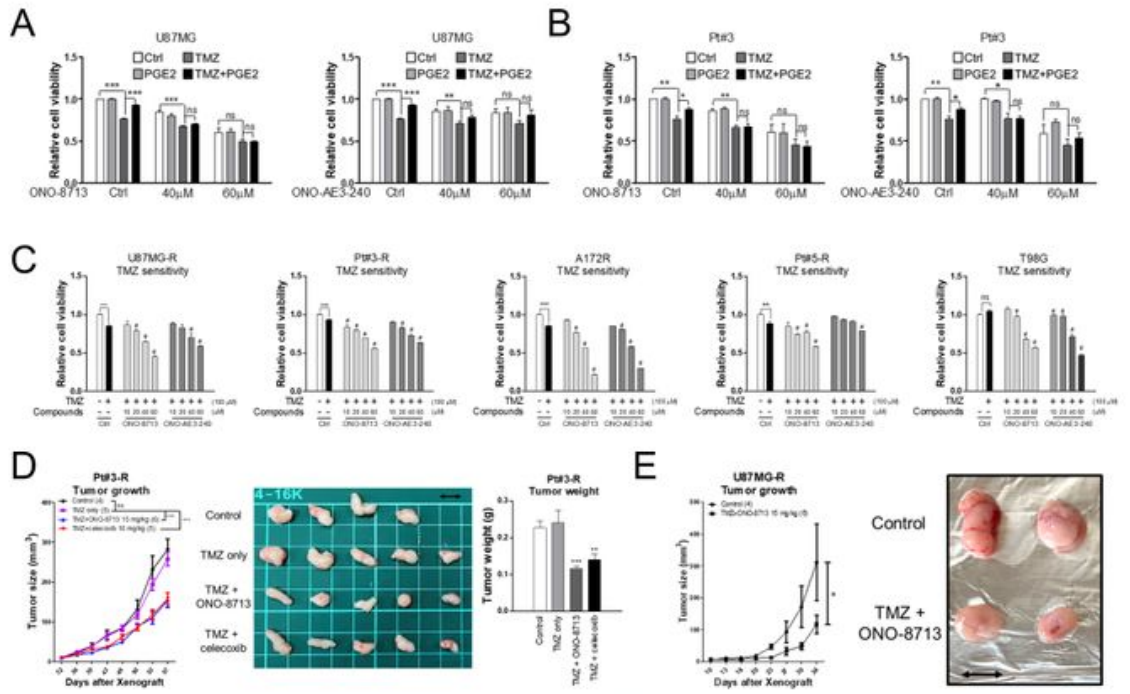

$\mathrm{F}$

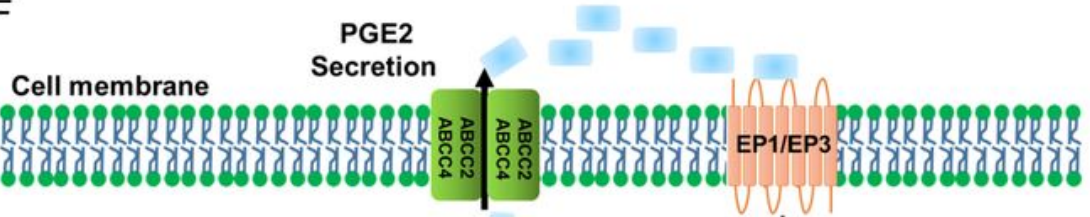

$\mathrm{AA}^{\mathrm{PGH} 2}-\rightarrow{ }^{\mathrm{PGE} 2}$

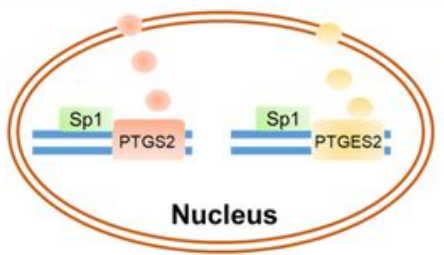

Mitochondrial fusion

ATP production 1
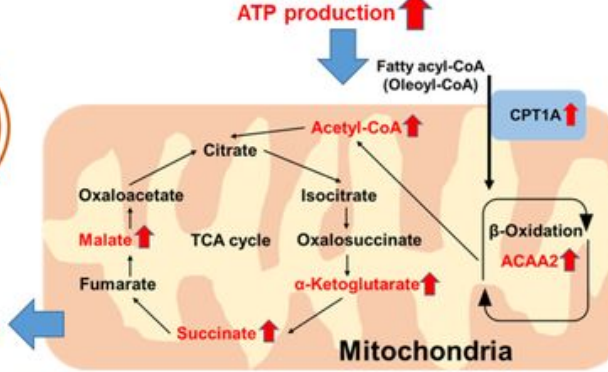

Figure 6 
Therapeutic effect of EP antagonists on TMZ-resistant glioblastoma. (A), (B) Effect of EP1 antagonist, ONO-8713, or EP3 antagonist, ONO-AE3-240, on glioblastoma cells. The cells were treated with ONO-8713 or ONO-AE3-240 in the presence of TMZ and PGE2 for $96 \mathrm{~h}$. Cell viability was determined via MTT assay. (C) TMZ-resistant glioblastoma cells treated with different compounds in the presence of TMZ for $96 \mathrm{~h}$. Cell viability was determined by performing MTT assay. (\# implies a significant difference compared with the TMZ-treated group). (D), (E) Effect of ONO-8713 or celecoxib on the growth of TMZ-resistant cellderived tumour in xenograft mouse model. The difference between the control and experimental groups was analysed by performing two-way ANOVA. The scale bar is $1 \mathrm{~cm}$. (F) Schematic representation of the working model.

\section{Supplementary Files}

This is a list of supplementary files associated with this preprint. Click to download.

- SupplementaryFig.1.docx

- SupplementaryFig.2.docx

- SupplementaryFig.3.docx

- SupplementaryFig.4.docx

- SupplementaryFig.5.docx

- SupplementaryTable1.docx

- SupplementaryTable2.docx

- SupplementaryTable3.docx

- SupplementaryTable4.docx

- SupplementaryTable5.docx

- SupplementaryTable6.docx 